\title{
Flow Generation by a Piezoelectric Fan
}

\author{
Myung Hun Oh, Heungsu Jeon, Minsuk Choi \\ Department of Mechanical Engineering, Myongji University \\ 116 Myongji-Ro, Cheoin-Gu, Yongin 17058, Republic of Korea \\ mchoi@mju.ac.kr
}

\section{Extended Abstract}

Since Toda [1] firstly introduced a vibrating flat plate, many researchers have conducted experiments to understand its effects on the heat transfer around a small heat source. Recently, it is relatively easy to make a vibrating plate with piezoelectric materials. When AC power is applied to a flat plate on which the piezoelectric material is deposited, the plate moves back and forth so that this movement can generate a small airflow to cool a small electric device. That's why a vibrating plate with the piezoelectric material is called as 'piezoelectric fan'.

Although most of researchers had been focusing on the heat transfer enhancement with a piezoelectric fan, Kim et al. [2] tried to understand the flow generated by the fan and measured two-dimensional velocity field using LDV (Laser Doppler Velocimetry). His experimental results clearly showed the formation of counter-rotating vortices by a piezoelectric fan. Choi et al. [3-5] conducted two-dimensional numerical simulations to obtain the flow around a piezoelectric fan and validated their results with the experimental data of Kim et al. [2]. Based on the numerical simulations, they investigated the exact moment of vortex separation from the fan and the effects of phase difference and distance between two fans on the flow generation. A few researchers succeeded in simulating the 3D flow around a vibrating plate, they adopted tetrahedral mesh with spring-based dynamic mesh and re-meshing techniques to implement the movement of the plate. Therefore, the simulated counter-rotating vortex was weaker than the experimentally measured one, even though the overall heat transfer rate agreed well between 3D simulations and the experiments.

This study is a continuation of the previous works [3-5] to calculate the 3D flow around a piezoelectric fan with minimizing the numerical dissipation caused by the mesh movement. Therefore, only hexahedral mesh was generated around the fan, and its movement was implemented by the spring-based dynamic mesh technique only without using the re-meshing. From the numerical results, it was found that the endwall has a significant effect on the flow around the fan. The clearance flow between the sides of the fan and the endwalls forms two alternating vortices. These vortices finally merged with the counter-rotating vortices generated at the plate tip, causing very complex flow near the endwall. These vortex motions make the axial velocity increase at the mid-span but decrease near the endwall. These flow structures around the piezoelectric fan have not been clearly resolved by tetra elements with the re-meshing technique in previous researches.

\section{Acknowledgements}

This research was supported by Basic Science Research Program through the National Research Foundation of Korea (NRF) funded by the Ministry of Education (No.2016R1D1A1B03932666).

\section{References}

[1] M. Toda, "Theory of air flow generation by a resonant type PVF2 bimorph cantilever vibrator," Ferroelectrics, vol. 22, pp. 911-918, 1978.

[2] Y.-H. Kim, S. T. Wereley and C.-H. Chun, "Phase-resolved flow field produced by a vibrating cantilever plate between two endplates," Physics of Fluids, vol. 16, pp. 145-162, 2004.

[3] M. Choi, C. Cierpka and Y.-H. Kim, "Vortex formation by a vibrating cantilever," Journal of Fluids and Structures, vol. 31, pp. 67-78, 2012.

[4] M. Choi, S.-Y. Lee and Y.-H. Kim, "On the flow around a vibrating cantilever pair with different phase angle," European Journal of Mechanics - B/Fluids, vol. 34, pp. 146-157, 2012. 
[5] M. Choi, C. Cierpka, Y.-H. Kim, "Effects of the distance between a vibrating cantilever pair," European Journal of Mechanics-B/Fluids, vol. 43, pp. 154-165, 2014. 